\title{
ÉTUDE DES STADES DE DÉBUT DE LA MÉIOSE CHEZ L'OVOCYTE DE LAPIN : COMPARAISON AVEC L'OVOCYTE HUMAIN
}

\author{
Monique DEVICTOR-VUILLET, J.-M. LUCIANI et A. STAHL \\ Laboratoire d'Histologie et Embryologie $I I$, \\ (Directeur: Professeur A. STAHL), \\ Faculté de Médecine, \\ 27, boulevard Jean-Moulin, \\ 13385 Marseille Cédex 4 (France)
}

\begin{abstract}
RÉSUMÉ
L'étude des stades de la méiose a montré l'existence constante chez l'ovocyte toetal humain d'un stade préleptotène de contraction chromosomique. Un tel processus est également observé chez la Lapine. L'étude comparative pratiquée révèle l'existence d'une variabilité dans l'intensité et l'étendue de ce processus de contraction chromosomique. Les stades ultérieurs de la méiose correspondent chez les deux espèces aux descriptions habituelles du processus méiotique.
\end{abstract}

Une étude cytologique de la méiose de l'ovocyte fotal humain nous a conduit à décrire pour la première fois dans le règne animal un processus de spiralisation et de condensation chromosomiques entre la dernière interphase préméiotique et le stade leptotène (STAHL et LUCIANI, I97I). Au maximum de leur contraction, les chromosomes sont suffisamment condensés pour apparaître comme 46 entités séparées. Une phase de déspiralisation survient alors, les chromosomes s'allongent et forment progressivement les filaments du stade leptotène.

La recherche de ce processus chez d'autres espèces animales nous a révélé des images tout à fait analogues chez la lapine.

Il semble, toutefois, exister des variations dans le degré de contraction chromosomique, celui-ci étant plus important pour 1'ovocyte fœetal humain.

L'analyse des stades ultérieurs de la prophase I chez le fœtus humain nous a permis de confirmer et de préciser, grâce à l'adoption de techniques cytologiques 
nouvelles pour ce matériel (LUCIANI et STAHL, I97I), les observations d'OHNo et coll. (Ig62). Celles-ci révélaient que les aspects morphologiques des divers stades de la prophase I, depuis le leptotène jusqu'au dictyotène, étaient conformes aux processus généraux de la méiose.

Le présent travail montre qu'il en est de même chez la Lapine et met aussi l'accent sur l'individualisation d'un stade préleptotène de contraction chromosomique.

\section{MATÉRIEL ET MÉTHODES}

Nous avons disposé pour cette étude de douze fœetus humains provenant d'avortements spontanés, régulièrement âgés de 2 mois à 9 mois.

L'étude chez la Lapine a été effectuée à partir de douze animaux âgés de 3 jours (I), 4 jours (2), 6 jours (2), 7 jours (2), ro jours (I), I7 jours (I), 24 jours (I) et 28 jours (2).

Nous avons choisi cette espèce, d'une part en raison de l'existence sur les planches d'ovogenèse dessinées par VON WINIWARTER en Igor de noyaux dont la morphologie correspond à celle de la phase de condensation maximale, d'autre part en raison du début post-natal de la méiose chez la Lapine (Maulton, rg67).

Nous avons pratiqué parallèlement des techniques histologiques et cytologiques aussi bien pour les ovaires humains que pour les ovaires de lapine.

Nous avons adapté pour l'étude cytologique des ovaires la technique déjà utilisée pour l'obtention des chromosomes méiotiques de l'Homme (Luciani et coll., r97 r).

Si cette méthode nous a donné d'excellents résultats dans le traitement des ovaires fœtaux humains, il n'en fut pas de même pour les ovaires de lapine.

Aussi, pour cette dernière espèce, avons-nous modifié la technique initiale de la façon suivante :

- l'ovaire prélevé est plongé, soit dans une solution hypotonique de chlorure de potassium pend ant 5 minutes à température ambiante, soit d'emblée dans une solution de méthanol/acide acétique glacial (2/I);

- la dilacération est effectuée au moyen de deux pinces droites Dumont dans le fixateur ; 8 minutes ;

- la totalité de la suspension obtenue est alors centrifugée à I ooo tours/minute pendant

- le culot est remis en suspension dans I $\mathrm{ml}$ environ d'acide acétique à $45 \mathrm{p}$. Ioo, fraîchement préparé, dont le temps d'action est maintenu jusqu'à la clarific $x$ tion des fragments que la dilacération, pourtant soigneuse, n'avait pu totalement éviter : cette durée est de 3 à 4 minutes ;

- les étalements sont alors réalisés soit sur lames glacées, soit sur lames sèches. Dans ce dernier cas, il est nécessaire d'ajouter quelques gouttes de méthanol à la suspension finale pour faciliter l'étalement.

\section{RÉSULTATS}

La séquence de développement depuis l'interphase préméiotique jusqu'au stade leptotène en passant par une phase de spiralisation puis de déspiralisation a déjà été décrite et illustrée pour l'ovocyte humain (STAHL, et LUCIANI, I97I).

Ultérieurement (LUCIANI et STAHL, I97I), nous avons distingué au cours du stade préleptotène trois phases : une phase de spiralisation, une phase de condensation maximale et une phase de déspiralisation.

Cette distinction peut être reprise pour le stade préleptotène de la lapine en raison des analogies morphologiques très grandes existant au même stade avec l'ovocyte humain. 


\section{A. - Le stade préleptotène}

a) La phase de spiralisation.

A la fin de 1'interphase préméiotique, le noyau présente un aspect granulofilamenteux provenant de l'existence simultanée de chromocentres et de filaments en voie d'individualisation (P1. I, fig. I et 2).

Le début du processus de spiralisation est caractérisé par l'individualisation plus marquée des filaments chromosomiques. Ceux-ci forment des boucles et des replis qui aboutissent à la constitution de masses denses, aux contours irréguliers. Chez les deux espèces, ces masses apparaissent plus précocément à un pôle du noyau (P1. I, fig. 3 et 4 ), à l'endroit où se trouve du matériel nucléolaire.

La poursuite $\mathrm{du}$ processus de spiralisation et de contraction chromosomiques se traduit par l'incorporation progressive des boucles et des replis. Ceci a pour corollaire l'augmentation de la taille des masses denses dont la polarisation s'est nettement accentuée (P1. I, fig. 5 et 6 ).

Les filaments chromosomiques continuent à se replier sur eux-mêmes. Le noyau est alors presque exclusivement constitué de masses denses (P1. II, fig. 7 et 8 ). Cet aspect peut être défini comme une phase de spiralisation moyenne.

La phase finale du processus de condensation est atteinte lorsque les masses chromosomiques deviennent pratiquement indépendantes les unes des autres (P1. II, fig. 9 et Io).

\section{b) La phase de condensation complète.}

Les chromosomes, alors au maximum de leur contraction, se présentent comme des entités séparées. Chaque chromosome apparaît constitué par une masse compacte de chromatine visible à la fois sur les coupes histologiques et sur les préparations cytologiques (P1. II, fig. II et I2). Seules ces dernières permettent de constater que le nombre de masses par noyau est toujours égal au nombre diploïde de l'espèce ou voisin de celui-ci.

La morphologie nucléaite de ce stade correspond à celle des noyaux à " prochromosomes " observés chez divers invertébrés (WIL,SON, I928).

Il faut remarquer que ces noyaux sont beaucoup plus rares dans les ovaires de lapine.

\section{c) La phase de déspiralisation.}

Le processus de déspiralisation est caractérisé par 1'apparition autour de chaque "prochromosome " de fins filaments qui s'échappent de la masse de chromatine et y reviennent après avoir décrit une boucle (P1. III, fig. I3 et I4).

Ainsi, chaque masse chromosomique se résout peu à peu en un feutrage de fibres chromosomiques rayonnantes (P1. III, fig. I5 et I6).

La fin du processus de déspiralisation conduit à la reconstitution des filaments chromosomiques qui présentent encore à ce stade des boucles et des replis (P1. III, fig. I7 et I8). Ce noyau ne peut être confondu avec celui de la phase de spiralisation précoce où les masses sont plus nombreuses, moins volumineuses et de contours plus irréguliers. De plus, la polarisation des chromosomes, présente au cours de cette phase de spiralisation, n'existe plus dans le noyau du stade préleptotène tardif. 


\section{B. - Les stades ultérieurs de la prophase}

Le noyau leptotène est constitué par l'enchevêtrement de longs et fins filaments parfaitement individualisés ( $\mathrm{Pl}$. IV, fig. I9 et 20). Le matériel nucléolaire semble moins visible, chez l'ovocyte de lapine; de même, on ne retrouve pas avec autant d'évidence la couronne d'hétérochromatine péri-nucléolaire que nous avons décrite chez l'ovocyte humain (STAHL et Luciani, I972).

La fin du stade leptotène est marquée par le début de l'appariement des chromosomes homologues et de la convergence des filaments vers un pôle du noyau.

Au fur et à mesure de la progression des appariements, la zone de convergence, nettement excentrée dans le noyau, devient de plus en plus évidente : les chromosomes du stade zygotène, étroitement pelotonnés, réalisent une masse compacte d'où s'échappent des anses chromosomiques (P1. IV, fig. 2I et 22).

Au stade pachytène, les appariements sont réalisés. Dans les deux espèces, les bivalents sont longs, fins, flexueux et présentent des rapports avec le matériel nucléolaire. Nous avons montré que dans l'espèce humaine ces rapports sont réalisés de façon préférentielle par les chromosomes acrocentriques (LUCIANI et STAHL, I97I).

\section{DISCUSSION}

Nous ne reviendrons pas sur les problèmes posés par l'interprétation des noyaux à " prochromosomes " et exposés antérieurement (STAHL et LUCIANI, I97I), si ce n'est pour souligner leur présence dans les zones superficielles des ovaires des lapines les plus jeunes. Comme dans les ovaires foetaux humains, ces noyaux se situent dans une zone où l'on s'attend précisément à trouver les formes de passage entre l'ovogonie et l'ovocyte au stade leptotène.

De plus, l'existence d'un stade préleptotène de condensation et de spiralisation semble être retrouvé chez un nombre croissant d'espèces.

La Lapine est le second mammifère chez lequel nous observons cette particularité de début de la méiose. Celle-ci existe aussi probablement chez la Caille et le Poulet (recherches en cours).

Parallèlement, WALTERS (I972) décrit un stade préleptotène de contraction chez Lilium longiflorum "Ace " et chez l'hybride Lilium " Enchantment ". Cet auteur étend ainsi à deux formes différentes de lys ses observations initiales faites en $197^{\circ}$ chez Lilium longiflorum " Croft ".

Mais WALTERS (1972) note une grande variabilité dans le degré de la contraction chromosomique : chez Lilium longiforum "Nellie White", seul un petit nombre de microsporocytes présente un processus de condensation et l'importance de celui-ci est bien moins grande que celle observée dans la forme "Croft ". A l'inverse, la condensation et la spiralisation chromosomiques semblent particulièrement prononcées chez "Ace " et chez " Enchantment ".

Nos observations nous conduisent aux mêmes conclusions : le processus de contraction chromosomique est beaucoup plus intense chez l'ovocyte humain que chez l'ovocyte de la Lapine. Chez cette dernière espèce, on observe, en effet, beaucoup moins 
de noyaux à "prochromosomes " : or, ceux-ci représentent pour nous l'état le plus complet de la spiralisation. Tout se passe comme si, une fois arrivés à une phase déjà avancée de la spiralisation (P1. II, fig. 8), les noyaux préleptotènes amorçaient le processus de déspiralisation devant les conduire au stade leptotène.

Enfin, bien que la signification exacte d'un tel processus de contraction chromosomique au début de la méiose soit encore inconnue, il n'en est pas moins utile d'explorer diverses espèces, tant animales que végétales, et de comparer les résultats obtenus.

\title{
REMERCIEMENTS
}

Nous remercions très sincèrement Mlle Arlette NAssI pour son aide à la confection des coupes histologiques et $M^{11}$ Noëlle Leguillou pour toutes les réalisations photographiques.

\author{
SUMMARY \\ COMPARATIVE STUDY OF THE FIRST STAGES OF MEIOSIS \\ IN RABBIT AND HUMAN OOCYTE
}

A period of preleptotene chromosome contraction was regularly observed in human fetal oocytes. This process is also observed in rabbit oocytes. A comparative study shows the variability of this preleptotene chromosome contraction. In the two species, the later stages of meiosis are identical through the general meiotic process.

\section{RÉFÉRENCES BIBLIOGRAPHIQUES}

Luciani J. M., Devictor-Vulllet M., Stahl A., I97I. Hypotonic KCl : an improved method of processing human testicular tissue for meiotic chromosomes. Clin. Genet., 2, 32-36.

Luciani J. M., Stahl A., r97r. Étude des stades de début de la méiose chez l'ovocyte fœetal humain. Bull. Ass. Anat., 151, 445-458.

Luciani J. M., Stahl A., I97I. Rapports des nucléoles avec les chromosomes méiotiques de l'ovocyte foetal humain. C. R. Acad. Sci., Paris, 273, 521-524.

Maulkon P., 1967. Cinétique de l'ovogenèse chez les mammifères. Arch. Anat. Microsc. Morph. Exp., 56, suppl., 125-150.

Ohno S., KLINGer H. P., AtKin N. B., I962. Human oögenesis. Cytogenetics, 1, 42-5I.

Stahl A., Luciani J. M., x97I. Individualisation d'un stade préleptotène de condensation chromosomique au début de la méiose chez l'ovocyte foetal humain. C. R. Acad. Sci., Paris, 272, 204 I-2044.

Stahl A., Luciani J. M., r972. Nucleoli and chromosomes : their relationships during the meiotic prophase of the human fetal oocyte. Humangenetik, 14, 269-284.

Walters M. S., 1970. Evidence on the time of chromosome pairing from the preleptotene spiral stage in Lilium longiflorum CROFT. Chromosoma, 29, 375-4I8.

Walters M. S., 1972. Preleptotene chromosome contraction in Lilium longiforum croft. Chromosoma, 39, 31 I-332.

Wilson E. B., I928. The cell in development and heredity. $3^{\mathbf{e}}$ ed. Mac Millan, New York, 1232 pages. Winiwarter H. von, Igor. Recherches sur l'ovogenèse et l'organogenèse de l'ovaire des mammifères (Lapin et Homme). Arch. Biol., Liège, 17, 33-I99. 
N. B. : Pour toutes les planches, les photographies situées d̀ gauche (chiffres impairs) représentent les ovocytes fotaux humains, celles situées à droite (chiffres pairs) les ovocytes de lapine.

\section{PLANCHE I}

FIG. I et 2

Noyaux granulo-filamenteux de l'interphase préméiotique $(\times 2600$ et 2300$)$.

FIG. 3 et 4

Noyaux au stade préleptotène précoce : début de condensation et de spiralisation chromosomiques. Noter les boucles et les replis (flèches) et la polarisation autour du nucléole $(n)(\times$ I 700 et 2000$)$.

FIG. 5 et 6

Noter l'accentuation de la spiralisation et l'accroissement des masses denses par incorporation progressive des filaments chromosomiques ( $X$ I 000 et 2600 ). 

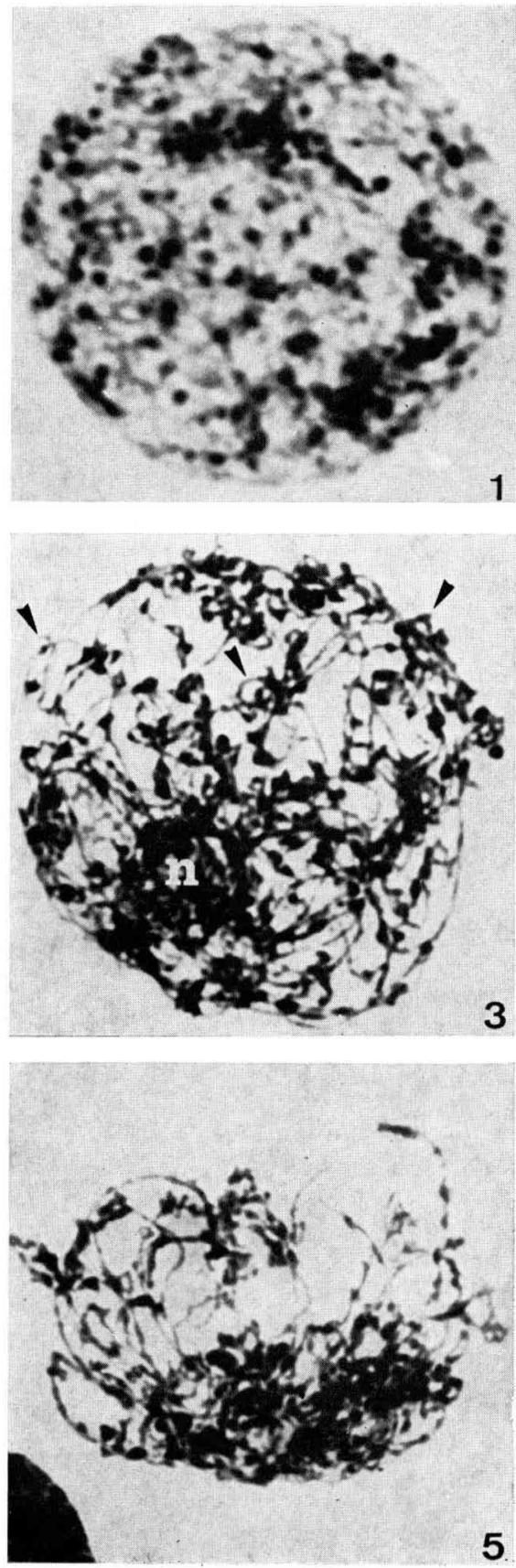
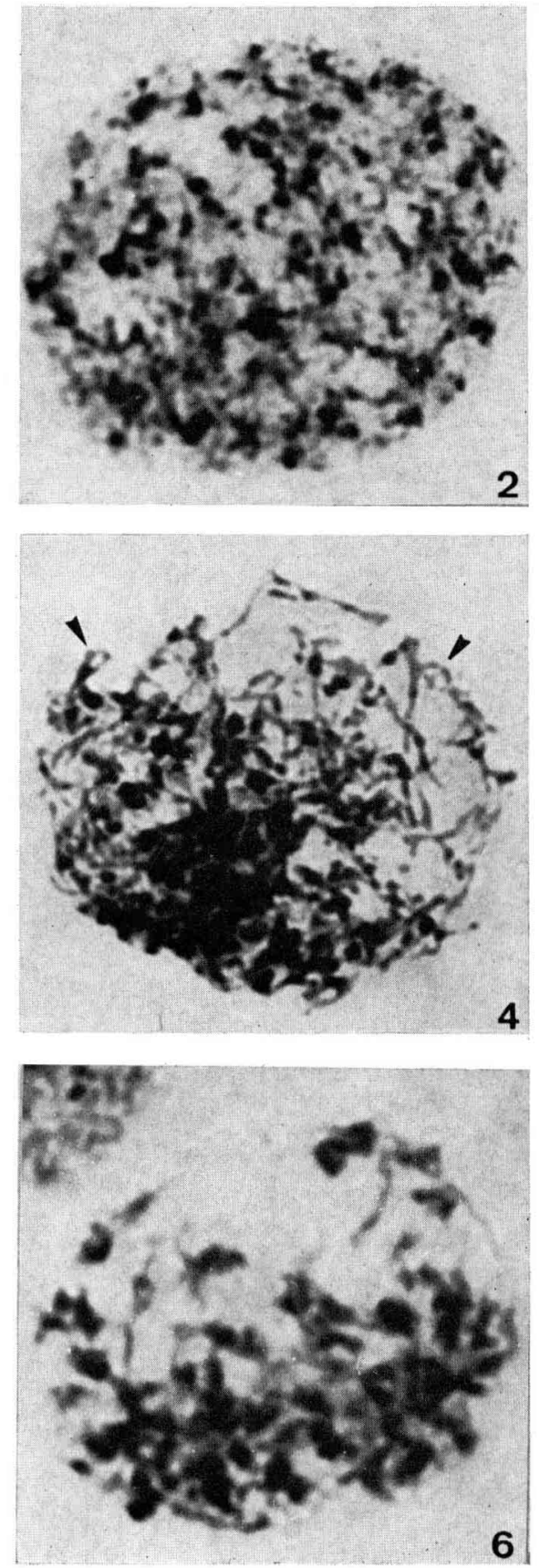


\section{PLANCHE II}

Fig. 7 et 8

Spiralisation et condensation moyennes : les noyaux sont presque exclusivement constitués de masses denses : persistance de quelques boucles non incorporées (flèches). $\times 1.800$ et 2.200 .

FIG. 9 et 10

Spiralisation et condensation avancées : les masses chromosomiques sont pratiquement indépendantes. $\times 1.400$ et 3.200 .

FIG. 11 et 12

Phase de condensation maximale : les chromosomes dont le nombre est voisin de la diploïdie forment des entités séparées. $\times 1.700$ et 4.000 . 
PLANCHE II
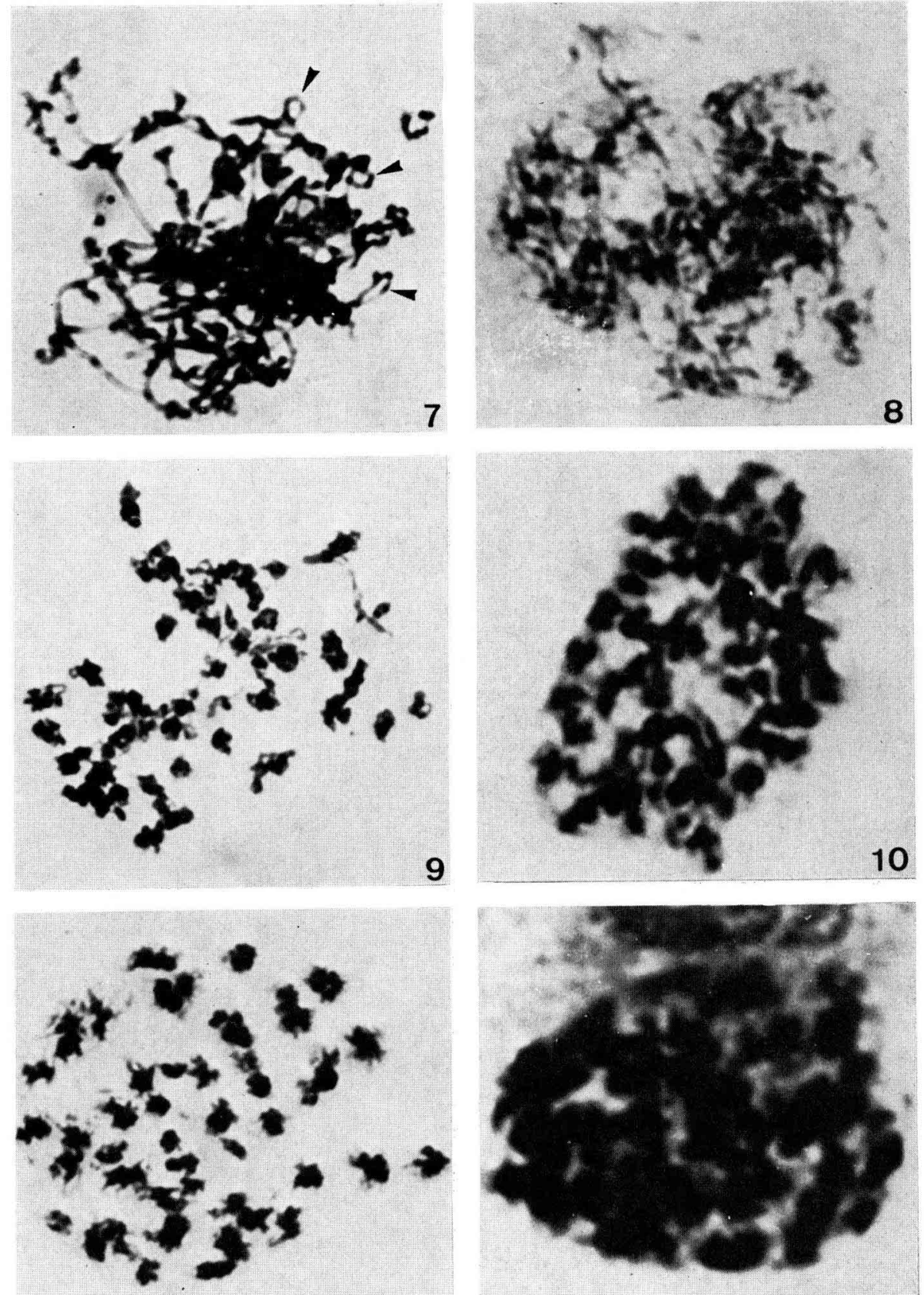


\title{
PLANCHE III
}

\author{
Fig. 13 et 14
}

Noyaux préleptoténes : début de déspiralisation. Apparition des filaments chromosomiques autour de chaque masse. $\times 1.800$ et 4.600 .

FIG. 15 et 16

Déspiralisation moyenne : existence d'un feutrage de filaments autour de chaque masse chromosomique. $\times 1.600$ et 2.600 .

Fig. 17 et 18

Stades préleptotènes tardifs : reconstitution des filaments chromosomiques dont certains présentent encore des boucles (flèches). $\times 1.400$ et 2.000 . 

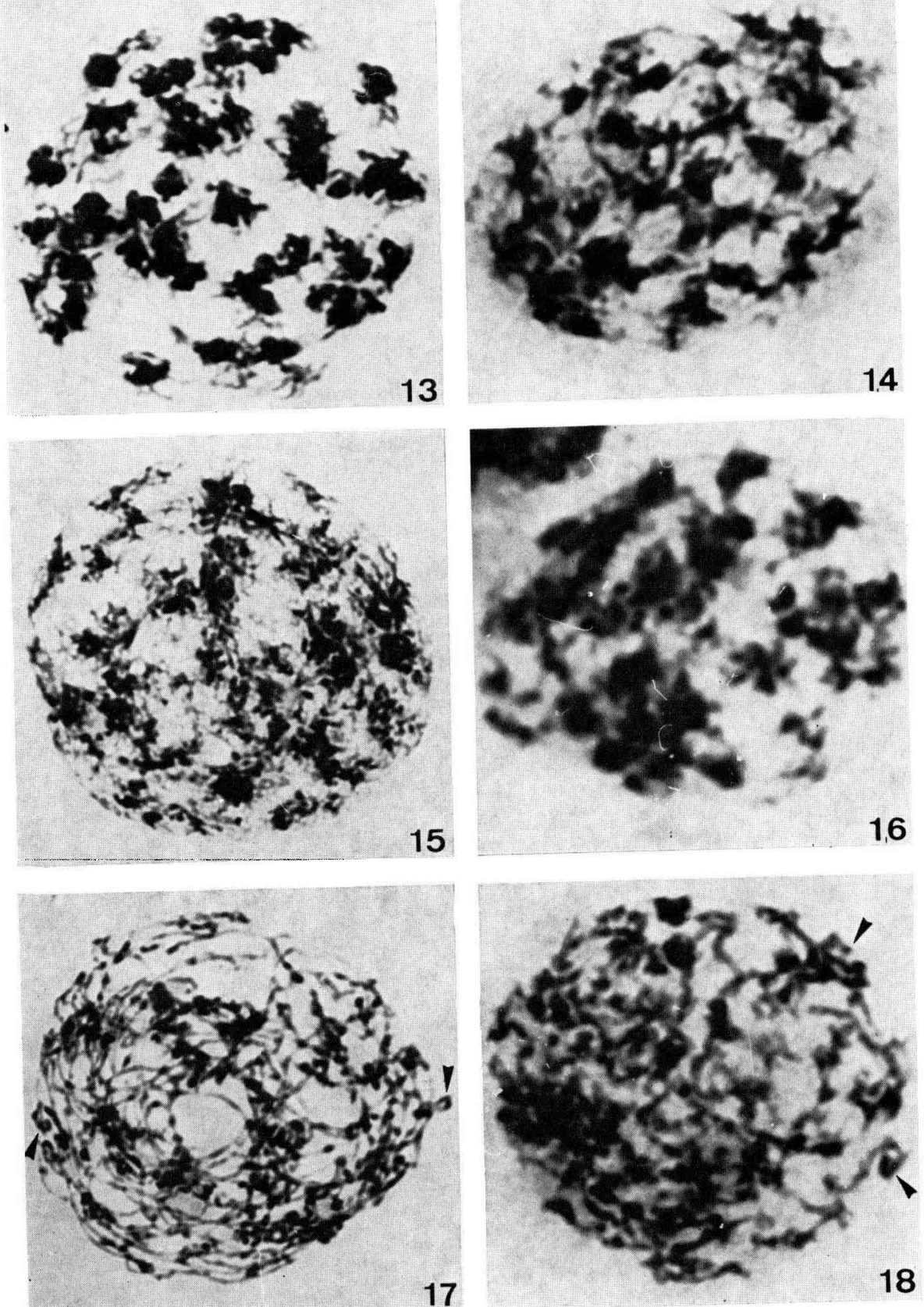

M. DEVICTOR-VUILLET, J.-M. LUCIANI et A. STAHL 


\section{PLANCHE IV}

\section{FIG. 19 et 20}

Noyaux leptotènes : noter chez l'ovocyte humain la couronne d'hétérochromatine péri-nucléolaire (n.). $\times 1.900$ et 2.300 .

Fig. 21 et 22

Noyaux zygotènes : appariements en cours de réalisation et polarisation des chromosomes. $\times 1.500$ et 2.700 .

$$
\text { Fig. } 23 \text { et } 24
$$

Noyaux pachytènes : noter les rapports nucléolaires de certains bivalents. $\times 1.000$ et 1.500 . 

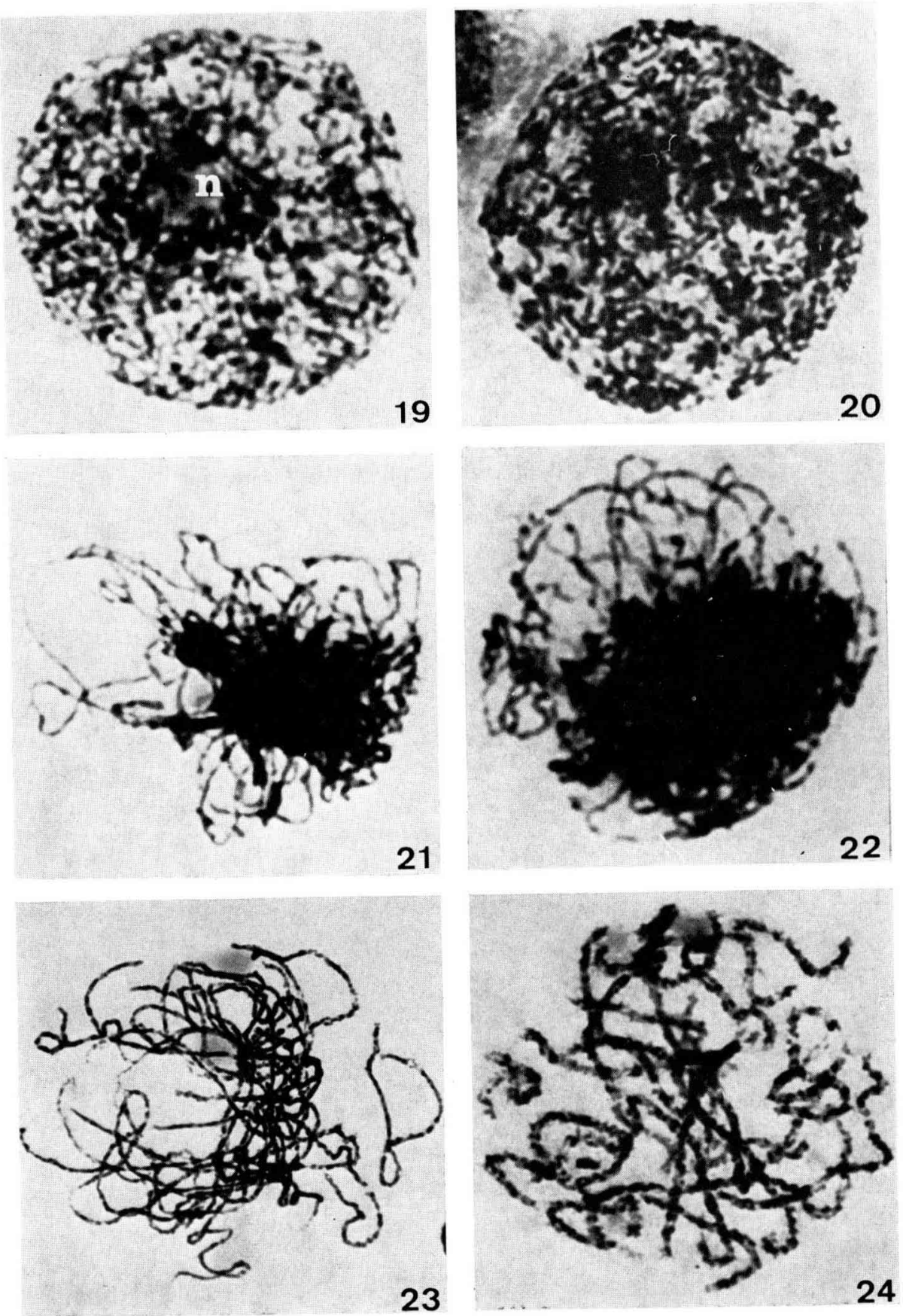

M. DEVICTOR-VUILLET, J.-M. LUCIANI et A. STAHL 